\title{
Autoevaluación del aprendizaje \\ en arquitectura
}

Self evaluation of the learning process
in architecture

\section{Aгq. Ana María Rugiero P.}

$<$ Resumen>

Se expone el fundamento teórico, metodología y conclusiones de la investigación realizada sobre los efectos de la autoevaluación del estudiante en su formación. Aplicada a Talleres de $1^{e r}$ y $2^{\text {do }}$ año de Arquitectura, permitió definir los requisitos y particularidades de la Autoevaluación Sistemática, nueva y eficaz estrategia didáctica.

\section{$<$ Abst ract $>$}

The following text contains the theoretical background, methodology and concl usions of a research on the effects of self eval uation on students during thei $r$ education.

<Palabras clave>

AUTOEVALUACIÓN / COMPETENCIAS / APRENDIZAJE AUTODIRIGIDO / METACOGNICIÓN

$<$ Key words>

SELF EVALUATI ON / TRAI NI NG FOR COMPETENCES / META COGNI TI ON / SELF LEARNI NG PROCESS

\section{Introducción}

La formación universitaria se ha visto exhortada en las últimas décadas a responder en forma innovadora, a los requerimientos de nuestros tiempos. Pero innovar en el ámbito universitario no resulta fácil, pues no cabe renunciar al alto nivel de exigencia que lo caracteriza. Además, la adecuación de los curricula a las nuevas realidades sociales y culturales -que sigue siendo recomendadamantiene una sujeción a los tiempos destinados a la formación, que no pueden ampliarse sino que requieren, más bien, ser reducidos a -al menos- optimizados.

Schön (1992) señaló el importante rol de la temporalidad en las carreras artísticas: "Realmente, nada es tan indicativo del progreso en la adquisición del arte como el descubrimiento que hace el estudiante del tiempo que ello lleva, tiempo para vivir a través del choque inicial cargado de confusión y misterio, olvidar las expectativas previas y comenzar a dominar la práctica del practicum; tiempo para vivir a través de los ciclos de aprendizaje implicados en cualquier tarea de diseño y finalmente tiempo para ir de atrás para adelante, una y otra vez entre la reflexión sobre la acción y la reflexión en la acción".

Así, la temporalidad del joven se ve distorsionada por un tipo de reflexión que, por lo general, siendo paralela al aprendizaje de la forma arquitectónica en sí misma, adopta la forma de introversión: no es controlada a nivel consciente, y en su devenir natural, resulta azarosa y errática.

Guiados por estas consideraciones hemos implementado la Autoevaluación Sistemática del Proyecto como recurso didáctico orientado a optimizar el tiempo de aprendizaje y a consolidar los nuevos conocimientos sobre la arquitectura, en la carrera que imparte la Facultad de Arquitectura y Urbanismo de la Universidad de Chile. Esta experiencia fue acompañada de una investigación ${ }^{1}$ metódica,

Proyecto: "Investigación Evaluativa del Aprendizaje Autoconstruido por Alumnos de $1^{\text {er }}$ y $2^{\text {do }}$ año de la Carrera de Arquitectura". Rugiero P., A.M., Investigadora Responsable; Apip, G.; Hirmas, A.; Y., Andrés, Coinvestigadores. Concurso FAU, 2002-2004. Facultad de Arquitectura y Urbanismo, Universidad de Chile. 
en el marco de determinar la incidencia de la autoevaluación en competencias propias de la autonomía del egresado, aspecto que entendemos consubstancial de la formación universitaria.

\section{Aspectos téricos relevantes}

El enfoque teórico de la investigación puede ser condensado aquí como la detección de una forma lógica común -en el nive conceptual más amplio y abstracto- entre la enseñanza en el Taller de Arquitectura y el desempeño laboral real. A tal efecto, citamos como referentes teóricos a Schön (1983; 1992) y Mabardi (2001). El primero, por cuanto desarrolló una epistemología de la práctica que se basa en el conocimiento en la acción, y la reflexión en la acción, acuñando la idea de un "prácticum reflexivo" cuyos rasgos son: el aprender haciendo, la tutorización antes que la enseñanza, y el diálogo entre el tutor y el estudiante sobre la mutua reflexión en la acción. Esto lo llevó a definir el currículum como "un contrato reflexivo entre el profesor y el alumno unidos por la indagación" y a encontrar en el Taller de Arquitectura un paradigma del prácticum. Schön se preguntaba: "iDe qué otro modo pueden los profesionales aprender (...) si no es a través de la reflexión sobre los dilemas de la práctica?".

Mabardi (2001) por su parte, sostuvo que la práctica del proyecto es la estrategia tradicional de la enseñanza de la arquitectura y que es factible ratificar su pertinencia en los tiempos actuales, optimizando los recursos que le son propios. Coincide así con Schön a atribuir validez y eficacia al aprender haciendo y al reconocer -él también- "los misteriosos fenómenos de la competencia y el arte de la práctica, y los no menos enigmáticos procesos por los que, en ocasiones, se adquieren este arte y esta competencia" (Schön, 1992)

Mabardi postula que la enseñanza de la arquitectura permite la formación en el proyecto y, a la vez, abre la posibilidad de una formación por el proyecto. Entiende que la primera es esencialmente profesional y conducente al dominio de la práctica del proyecto, y que la segunda es esencialmente universitaria pues debería conducir a una verdadera autonomía en todos los campos. El proyecto debería ser, según esto, el nudo de articulación de múltiples enseñanzas. Y el referente de la enseñanza, desde esta perspectiva, es también aquí, la práctica del profesional arquitecto, dado que el Taller de Diseño Arquitectónico se estructura según una serie cíclica de ejercicios proyectuales.

Mabardi establece un paralelismo entre las trayectorias del profesional, del docente y del alumno, subrayando en el primer caso, la reflexión sobre lo hecho, entre encargos, que permite al profesional reformular la voluntad de arte y la postura de actuación. Y exhorta a incluir similar revisión de lo hecho en la docencia y en el aprendizaje, para que profesores y estudiantes sean, también ellos, reflexivos; para que respondan con mayor propiedad a los requerimientos que la sociedad plantea hoy día a la formación universitaria.

Atendiendo postulados como los citados, y ante la evidencia de que la enseñanza de la arquitectura suele prescindir de la reflexión "entre proyectos", junto a dos profesores de Taller de Diseño de $1^{\text {er }}$ y $2^{\text {do }}$ año, nos propusimos incorporar la autoevaluación del alumno tras cada ejercicio proyectual, y determinar luego, científicamente, su incidencia en el objetivo último de la formación: la autonomía del egresado. El modelo de la enseñanza adquiere así la continuidad estructural inherente a la trayectoria profesional real -su forma lógica- y la reflexión entre proyectos se canaliza en lo que, desde nuestro enfoque, es su equivalente formativo: la autoevaluación (Figura 1).

\section{Aspectos metodológicos}

La innovación planteada en nuestro proyecto fue concebida como estrategia que conjuga docencia e investigación a la vez. En lo docente, consistió en proponer al alumno la autoevaluación -en el caso de trabajos individuales- o la coevaluación, para los ejercicios realizados en grupo. Se aplicó a partir del Semestre II de la carrera, y se usaron cinco formatos: para $1^{\text {er }}$ año, la Autoevaluación Referencial y la Autoevaluación de Niveles Cognitivos; para ambos niveles de la formación, la Coevaluación del Trabajo Cooperativo; y para $2^{\text {do }}$ año: la Autoevaluación Abierta y la Incidente en la Nota (Cuadro 1).

Los ítem de cada autoevaluación fueron referidos a los objetivos del ejercicio realizado y centrados en la propuesta personal del estudiante, interrogando sobre los criterios disciplinarios en juego, las etapas del procedimiento, etc. Para los ejercicios en grupo, se solicitó coevaluar la presencia de actitudes -propias y de sus compañeros-que favorecen la armonía y el logro de los objetivos del trabajo conjunto².
Esta innovación, válida en sí misma por su carácter formativo, pasó a constituirse en el tratamiento experimental de la investigación pedagógica realizada. El diseño de investigación fue del tipo cuasi experimental, longitudinal, con grupo de control y posprueba únicamente. La variable independiente -autoevalución- se manipuló en dos grados: presencia y ausencia; y la variable dependiente -autonomía- se operacionalizó según cuatro indicadores: capacidad crítica, objetividad metodológica, trabajo en equipo y participación activa en el propio aprendizaje.

La muestra estuvo conformada por grupos naturales, dos experimentales y dos de control por cada nivel, correspondiendo los cursos de $1^{\text {er }}$ año al año lectivo 2002 -con 40 alumnos cada uno- y los de $2^{\text {do }}$ año al 2003, conformados inicialmente por 27 alumnos -el grupo experimental-y por 25 -el de control-. La conformación de los grupos varió a lo largo del período; no obstante, se mantuvo la equivalencia de los grupos, ratificándose la confiabilidad de los grupos naturales respecto a las diversas variables que inciden en la validez interna (Hernández S., 2001)

Para la posprueba se utilizó un cuestionario autoaplicado, con cuatro preguntas y alternativas de grado, y se pidió justificación abierta de la alternativa elegida respecto de las tres primeras. Se realizó además una entrevista grupal semi estructurada -sesión en profundidad-como técnica complementaria de recolección de datos, y como medio para conocer el punto de vista de los estudiantes sobre la experiencia de autoevaluar sus proyectos.

\section{Resultados obtenidos}

Los resultados indican una correlación positiva entre el ejercicio de la autoevaluación y el incremento de la capacidad crítica, la objetividad metodológica y una predisposición más alta hacia el trabajo en equipo. Respecto a la participación activa en el propio aprendizaje, los resultados indican un comportamiento particular del estudiante de arquitectura que llevó a aportar criterios para replantear los presupuestos considerados en el estudio.

La experiencia implementada, tomada en su conjunto, permite recomendar la aplicación generalizada de lo que denominamos Autoevaluación Sistemática del Proyecto (ASP). Los requisitos que definen esta nueva

Para mayor información al respecto, remitimos a: Rugiero, A.M. et. al. Evaluación del aprendizaje autoconstruido. Motivando la autonomía del universitario. Santiago, Chile: Ediciones FAU, Universidad de Chile, 2006. 
construcción teórica (Cuadro 2) permiten maximizar su potencial como estrategia fundamental de una formación universitaria pertinente, acorde al contexto sociocultural actual, y con absoluto respeto a la libertad de cátedra.

Así mismo, la implementación de la ASP puso de manifiesto la efectividad de mecanismos que desde la sicología cognitiva se destacan como substanciales a los procesos de aprendizaje, sean éstos formales 0 autodirigidos. En lo fundamental, la ASP contribuye a la toma de conciencia, por parte del estudiante, de su propia identidad (Botella, 2000); favorece el desarrollo de procesos proactivos de atribución de significado a la experiencia (Mahoney, 1991), y permite ir acuñando la propia teoría personal sobre el desempeño gratificante, privilegiándolo, y desechando lo que aleja de los propios intereses y objetivos (Kelly, 1991).

Definimos la Autoevaluación Sistemática del Proyecto como la reflexión del estudiante sobre el propio desempeño, efectuada al término de cada uno de los ejercicios proyectuales contemplados en el ramo eje de la carrera, desde los primeros años de la formación, reflexión que le permite observar sus propios tropiezos y logros, las actitudes y modos de proceder que favorecieron, en mayor o menor medida, la construcción de nuevos conocimientos.

En términos generales, pudo determinarse que la Autoevaluación Sistemática tiene en sí misma, un carácter formativo; que contribuye a ejercitar en el desempeño profesional real, y a catalizar la construcción de conocimientos disciplinarios. Es una herramienta eficaz
Figura 1: Forma lógica de la enseñanza del proyecto

Trayectoria del Profesional

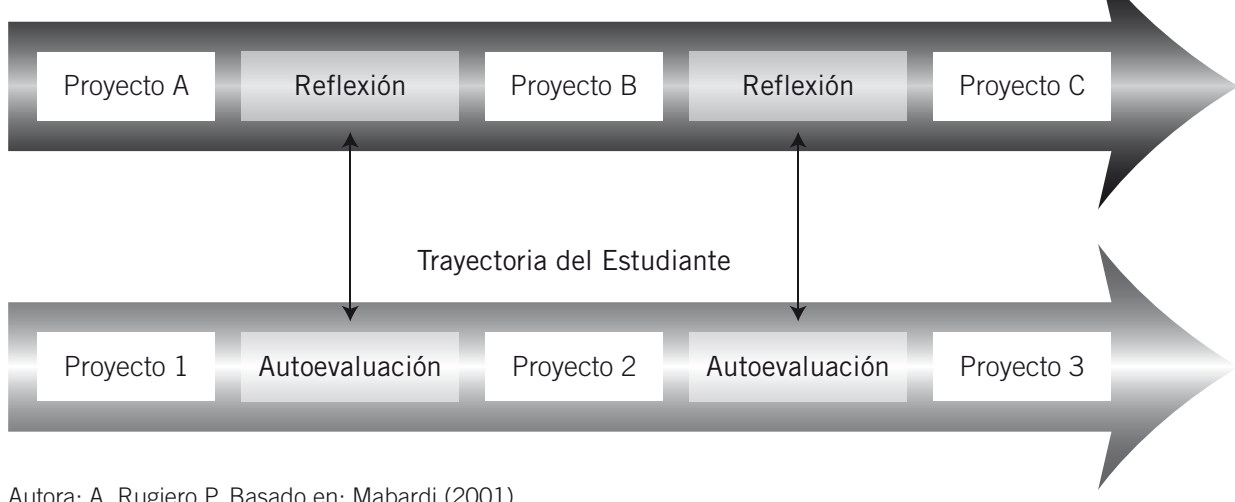

para fortalecer la autonomía del universitario, permitiéndole hacer extensivas a otros ámbitos de desempeño, las habilidades y destrezas inherentes a la profesión.

Las opiniones de los estudiantes que autoevaluaron sus proyectos confirman que la sistematización de esta estrategia didáctica refuerza la autoconfianza, y consolida su capacidad crítica y de autocrítica, respetando los procesos afectivos implícitos en todo proceso de aprendizaje. Cabe citar, como ejemplo, opiniones relativas a la conciencia sobre el propio aprendizaje y a la experiencia de autoevaluarse. La autoevaluación:

- "...Me sirvió para aprender de eso en que me equivocaba y seguir creciendo".

- "He aprendido a darle más énfasis al análisis previo..".
- "Aprender de mí mismo me ayuda a saber cómo seguir".

- "Esos espacios de reflexión son fundamentales". puede autoevaluarse mejor y ser más objetivo también".

- "Uno parte autocriticándose después de los proyectos, pero cada vez te vas acercando más al punto "cero": ¿Estoy partiendo bien, con este proyecto? ¿Es este el enfoque; el emplazamiento es correcto? Implica seguridad pero también (...) ponerse en la inseguridad".

- "Con el tiempo, me ha dado la posibilidad de ver el proceso, mientras estoy haciendo mi proyecto, y de ver desde fuera lo que
- "A medida que uno sabe un poco más,

\section{Cuadro 1: Recomendaciones/Requisitos de la autoevaluación sistemática del proyecto}

\section{Recomendaciones para el diseño de instrumentos}

- Derivar los criterios y categorías de análisis de indicadores explícitos, para dar coherencia al diseño de instrumentos, codificación de datos, logro en cada indicador.

- Referir la autoevaluación al ejercicio académico, en términos de procedimiento y/o de resultado.

- Interrogar sobre los objetivos del ejercicio y/o del nivel de formación.

- Emplear los conceptos privilegiados en el planteamiento del ejercicio, su desarrollo y correcciones.

- Recurrir a formas verbales reflexivas, y en primera persona.

- Referir las coevaluaciones a las actitudes deseables para un armónico y eficiente trabajo en equipo.

- Incluir respuestas abiertas, ya sea para justificar una alternativa elegida, o para expresar opiniones sobre conceptos o indicadores más generales.

- Dar progresividad a las autoevaluaciones en número e importancia de su ítem; diseñar un micro programa paralelo al de ejercicio de la asignatura.

- Recuperar siempre los formularios y devolverlos después, a fin de procesar los datos y posibilitar su archivo, por parte del estudiante. Las anotaciones del equipo docente, de incluirse, deberán ser respetuosas de las apreciaciones del alumno.

- Consensuar con los profesores del mismo nivel y niveles sucesivos de la formación, la secuencia y progresividad de las autoevaluaciones, a fin de graduar la complejidad y eficacia de la experiencia autoevaluativa. El estudiante debería transitar desde la autoevaluación referencial -de interés para él mismo- a la autoevaluación de respuestas abiertas y luego a la autoevaluación incidente, con aumento progresivo del porcentaje de participación en la nota final. 


\section{Cuadro 2: Glosario}

Autoevaluación referencial

toevaluación de niveles cognitivos

Autoevaluación abierta

Coevaluación
De interés exclusivo para el estudiante, y recomendable en especial para el $1^{\text {er }}$ año de la carrera. Culminando el ejercicio, antes o después de la entrega de calificaciones -según decisión del equipo docente- se entrega un cuestionario, se recoge - para procesar los datos- y se devuelve a la brevedad para que sea archivado, sin otro comentario que los de interés general, para todo el curso. Si la programación contempla más de tres autoevaluaciones, esta modalidad admite anotaciones o comentarios personalizados, aunque deberán ser no invasivos, es decir, respetuosos de las apreciaciones del alumno.

Recoge la asociación de la creatividad a los niveles cognitivos (Letelier, 2001). Interroga sobre el grado de complejidad cognitiva logrado: conocer, comprender, aplicar, analizar, evaluar, y/o sus equivalentes.

Recomendable para el $2^{\text {do }}$ año de la carrera, consiste en la entrega de una pauta referida a logros y fracasos respecto de los objetivos del proyecto y/o del nivel de la formación. También en esta modalidad corresponde procesar los datos obtenidos a partir de las respuestas abiertas, preferentemente según el indicador: "capacidad crítica".

Autoevaluación incidente Consiste en entregar al estudiante la pauta de corrección que usará el equipo docente, solicitando "calificar" cada ítem a evaluar, y dar al promedio global resultante una ponderación que incida en la nota final. Se recomienda aplicarla sólo a partir del último proyecto de $2^{\text {do }}$ año, y para $3^{\text {er }}$ y $4^{\text {to }}$ año. El porcentaje de incidencia en la nota final deberá ser progresivo, en un rango de entre el $10 \%$ y el $20 \%$.

Evaluación triangulada entre: el propio alumno, sus compañeros de grupo y equipo docente. Orientada a hacer explícitas la actitudes que permitan optimizar el trabajo en equipo, a éstas debe referirse los ítem. Su objetivo es que el estudiante conozca el comportamiento "ideal" y evalúe su accionar y el de sus compañeros en esa perspectiva. Conviene plantear en mesa redonda el análisis "amigable" de casos en que se dan mayores divergencias entre los integrantes del grupo y/o con el equipo docente, orientado sobre cómo y por qué conviene modificar determinadas actitudes.

Son aspecto observables y medibles que hacen operativa una variable (autonomía). Su formulación se desprende del marco teórico acordado por cada equipo docente. estoy realizando (...) ir mejorando en cuanto al manejo del proyecto".

- "Me ayudó a crear mi propio método de estudio-diseño-proyectación".

- "La autocrítica da la seguridad para pararse frente a un proyecto y saber que está bien (...) porque uno tiene fundamentos fuertes.."..

- "Es fundamental que esta experiencia de autocorregirse se haga desde el proyecto número uno".

\section{Reflexiones finales}

La autoevaluación está siendo propuesta entre las recomendaciones que surgen de las ciencias educativas, y puede haber sido implementada entre otras tantas innovaciones ensayadas, pero sin que se haya dejado constancia, antes de ahora, sobre su efectividad y pertinencia para potenciar aprendizajes significativos. Es en relación a algunos de esos intentos que he podido conocer, que me permito señalar la Autoevaluación Sistemática del Proyecto como una construcción teórica particular, nueva y en verdad eficiente para consolidar nuevos aprendizajes.

A los requisitos que se exponen en la Tabla 1 como propios de la ASP, cabe agregar las siguientes consideraciones globales:
- La ASP no se dirige a los contenidos cognitivos de la enseñanza. Los ramos teóricos sí los evalúan y la evaluación docente del proyecto muy probablemente no pueda excluirlos. Toda autoevaluación "cognitiva" es válida, pero no es una ASP.

- Es fundamental a la ASP la apelación a lo metacognitivo (Guzmán, 2001); es decir: al conocer cómo yo conozco. Se trata de una verdadera reflexión del estudiante, que contempla, observa y juzga su desempeño.

- Por lo anterior, cobra sentido el carácter referencial de las primeras autoevaluaciones. El alumno debe sentir que la propuesta que le hace el cuerpo docente está inserta en una estrategia formativa, y responder seria y responsablemente aún cuando no sea una actividad calificada.

- Dado que la ASP tiene por objetivo la construcción de aprendizajes "externos" al proyectar en sí mismo (la "estructura" del desempeño real; el pensamiento crítico; la autonomía) no cobra importancia el desajuste (esperable) entre la evaluación docente y la autoevaluación del estudiante. Tal coherencia recién adquiere relevancia al implementar la modalidad "Incidente" -que se recomienda reservar para el último proyecto de $2^{\text {do }}$ año, con un porcentaje mínimo (10\%) de participación en la nota-. Y para entonces, el estudiante habrá avanzado lo suficiente -en términos de autocrítica y de aprendizaje de la disciplina-como para ejercer esta opción con un mínimo desajuste.

- La retroalimentación de la experiencia de autoevaluarse pasa, en el marco de la ASP, por la devolución de las pautas y la socialización (comentario o debate) de los recursos que un profesional pondría en juego para mejorar su desempeño en aspectos aún deficitarios en el grupo-curso.

- Reiteramos el respeto absoluto a las apreciaciones de los estudiantes -que no impide pero tampoco impone el diálogo con el equipo docente y la fundamentación de juicios- diálogo que, de darse, ha de ser siempre paralelo a la plena validez de la evaluación docente.

- Por último, en relación a los recursos que demanda la ASP, es importante que la revisión y procesamiento de las pautas y formularios no graven el trabajo académico del cuerpo docente; por ello se incluye como rasgo diferenciador de la ASP la existencia del "ayudante / investigador" externo al aula. A la luz de lo ya avanzado, la aplicación de la ASP a nivel de la Escuela o Facultad, debería ser brindado por un grupo de académicos que tendría la función de dar continuidad y dinamismo a esta nueva estrategia pedagógica que se recomienda aquí. 